\title{
Comparison of oncologic outcomes of extrahepatic cholangiocarcinoma according to the location of tumor: Perihilar and distal bile duct cancer
}

\author{
Jung Min LEE, Hee Ju SOHN, Jae Seung KANG, Yoo Jin CHOI, Youngmin HAN, Hongbeom KIM, Jin-Young JANG* \\ Departments of Surgery and Cancer Research Institute, Seoul National University College of Medicine, Seoul, Korea
}

Introduction: Extrahepatic cholangiocarcinoma is distinguished into perihilar cholangiocarcinoma (pCCA) and distal cholangiocarcinoma (dCCA). There were few comprehensive studies including both subtypes while studies for each subtype were conducted separately due to different staging system and management. This study was aimed to compare oncologic outcomes between pCCA and dCCA and to clarify prognostic factor of each subtype.

Methods: Between 2001 and 2017, patients who underwent surgery for pCCA and dCCA were enrolled. We reclassified T stage to tumor extent as confined to or beyond bile duct (BD). In survival analysis, we performed stage matching based on extent of tumor and lymph node (LN) metastasis.

Results: Total 680 patients were enrolled, of whom 295 patients were diagnosed as pCCA and 385 patients as dCCA. R0 resection rate was higher in dCCA ( $77.3 \%$ vs. $89.9 \%, p=0.001)$. Tumor confined to BD were more common in pCCA $(61.7 \%$ vs. $37.7 \%, p=0.001)$. In survival analysis, 5 -year survival rate was higher in dCCA $(30.8 \%$ vs. $47.8 \%, p=0.001)$. After stage matching, dCCA still showed better survival outcomes (confined to BD, LN (-): 5 YSR $47.1 \%$ vs. $64.3 \%, p=0.001$; confined to $\mathrm{BD}, \mathrm{LN}(+): 22.0 \%$ vs. $35.0 \%, p=0.825$; beyond BD, $\mathrm{LN}(-): 21.9 \%$ vs. $49.8 \%, p=0.001$; beyond BD, $\mathrm{LN}(+): 9.6 \%$ vs. $26.9 \%$ vs., $p=0.050)$. The preoperative CA19-9 and tumor extent were prognostic factors for pCCA. Age, LN metastasis, differentiation and perineural invasion were prognostic factors for dCCA. R0 resection was prognostic factor for both subtypes.

Conclusions: dCCA showed better survival outcomes than pCCA. The prognostic factors between pCCA and dCCA were different as distinct entities. 